\title{
Retail 4.0 - Digital Customer and Retailer Feedback to Garment Development
}

\author{
Franziska Moltenbrey* and Dr. Thomas Fischer \\ German Institutes for Textile and Fiber Research, Germany
}

*Corresponding author: Franziska Moltenbrey, Department of Management, German Institutes for Textile and Fiber Research, Germany.

Received Date: April 16, 2021

Published Date: May 04, 2021

\begin{abstract}
Digital technologies open new possibilities ranging from integrated virtual product development to the direct involvement of customers and retailers through apps and collaborative virtual reality (VR) or augmented reality (AR) solutions. This requires end-to-end, flexible and modular solutions, especially in the retail environment.

In the Retail 4.0 research project, modular scalable software solutions were developed that enable the prompt transfer of feedback from retailers on garment development to the manufacturer. Thus, customers are directly involved on the basis of a new, digital VR/AR shopping experience. For example, the real shopping environment at the point of sale is additionally extended by a virtual solution to visualize garments on avatars via AR. In the B2B sector, clothing retailers and manufacturers exchange information about prototypes and future collections in a virtual showroom with the help of VR glasses.
\end{abstract}

Keywords: Digitalization; Fashion industry; Retailing; Collection development process; Virtual reality; Augmented reality; Virtual product development; Point-of-sale; Shopping experience; Fit

Abbreviations: VR = Virtual Reality; AR = Augmented Reality; POS = Point-of-Sale; SME = Small and Medium sized Enterprises; B2C $=$ Business-toConsumer; B2B = Business-to-Business; DITF = Deutsche Institute für Textil- und Faserforschung (German Institutes for Textile and Fiber Research Denkendorf); R\&D = Research and Development

\section{Introduction}

In the course of the digital transformation, the apparel industry is currently undergoing a fundamental structural change. Short production cycles, outsourced production sites and increased competition due to an increasingly demanding customer base are just some of the many challenges that the industry is facing. They require novel, digital solutions at the POS and in the garment development process in order to involve retailers and customers directly in product development at the manufacturer [1].

The paper is organized as follows: Section 1 describes the problems and challenges that arise in the course of digital transformation in the apparel industry. Section 2 reviews the state of the art of available technologies in the retail industry, then Section 3 presents new, digital solutions, especially for VR/AR, that consider both the interaction of the customer (B2C) and the integration of the manufacturer and retailer (B2B) in the process. Section 4 provides an outlook.

\section{Discussion}

\section{The garment industry facing change}

In the age of Industry 4.0 and e-commerce, SMEs in particular are often under pressure. Many companies have not yet implemented the digital transformation and need solutions to differentiate 
themselves from the competition through individualized service offerings [2]. Digital technologies open up numerous new opportunities for the apparel industry, from integrated virtual product development to the direct involvement of customers and retailers. At the moment, however, support for such processes in this environment is inadequate [2]. For SMEs in the textile and apparel industry, however, the implementation of digital measures, which are accompanied by numerous challenges, is not trivial. In addition to short production cycles and outsourced production sites, companies are also confronted with the increasing trend toward individualization and increasingly demanding customers [3]. Current processes are dominated by traditional value creation structures with a separation of development and sales. In addition, customers are not sufficiently integrated and are decoupled from the design and development process. Although feedback to the retailer or manufacturer takes place in the form of sales figures or evaluations of products, individual and product-specific approaches to body and fit are not taken into account. Targeted development and reliable estimation of demand based on these surveys is very difficult - shortages or overproduction are the result [1]. However, the future of the fashion industry is digital and integrated. For this reason, end-to-end, flexible and interactive solutions, especially in the retail environment, are needed to survive in the market and to react quickly to trends.

\section{State of the art}

The massive proliferation of mobile devices such as smartphones, tablets, wearables and head-mounted displays, nationwide broadband Internet coverage, and the continuous increase in available computing power are yielding great potential in terms of virtualization and networking of knowledge-intensive services with other innovation related processes. Consequently, the demand for innovative forms of interaction, new types of user experiences and the dynamic and demand-oriented provision of the services required for this purpose is growing steadily. This applies to both, the business and the consumer environment [4].

Digital concepts are currently establishing themselves in the area of product development for apparel manufacturers. Looking at the entire process of apparel creation, from concept development, design and pattern making to sample creation and subsequent production and retail positioning, innovative manufacturers are now using 3D simulation tools for the first time to create virtual apparel in the area of product development. Retailers, on the other hand, need solutions that are technically simple, flexible and robust to use without high upfront investments. Due to the required technical infrastructure, the continuous use of this virtual clothing models, even as a replacement for sales samples and in the retail trade, is therefore only possible to a limited extent and with great effort [4].

Collaborative virtual environments are successfully used, for example, in the automotive sector or for planning factories. Highly specialized systems, usually costing several 100,000€, are used for this purpose [5]. For the SME-dominated textile and apparel industry as well as the German retail sector, these system costs represent a major barrier. With the rapid development in head-mounted displays in recent years [6], inexpensive yet powerful systems are now available. Smartphones and tablets will play an important role in this context in the future. As a result, the barriers to entry for the required technical infrastructure are falling.

In general, shopping as an interactive experience is considered one of the major future trends [7]. The first attempts at interactive sales settings with mixed reality solutions [8] such as the Microsoft Hololens are currently taking place in the automotive sector [9]. However, interactions are mostly limited to product configuration and viewing. Use of interactive sales settings as a feedback channel to give voice to customers and retailers is not taking place. In the future, both the manufacturing of products and the entire collection development process will be geared to customers $[10,11]$.

A digital interaction option during collection duplication, i.e. the sale of collections by representatives to customers, is the digital showroom - a virtual place where collections can be viewed virtually, independent of time and space. The approaches from the BMBF project "Retail 4.0 - Virtual Retailer feed-back and Augmented Shopping as a knowledge-intensive, digital service in the development and sales process", replace the representative sample collections with AR/VR solutions and thus make a large part of the sample production superfluous. With the solution envisaged there, direct feedback from customers or retailers can be digitally captured by the representatives and fed back directly into the collection development process. This saves the time-consuming procedure of handing over the collection (via photo creation, manual uploading of files, entering in various tools) as has been practiced in the process to date (AiF Digital Collection Development). In the following section, two developed scenarios are explained in detail [4].

\section{Retail 4.0-Scenarios}

The established value chains in the apparel industry need new types of digital solutions to focus on the customer. This requires tools to support both, the integration of customers and retailers in the collection development process. For this purpose, new solutions for virtual and augmented reality in the retail industry were developed, in which feedback processes from customers to retailers to manufacturers are digitally and virtually supported. Two different B2C and B2B scenarios were developed that extend the existing technologies already used today in the collection development and manufacturing process.

B2C - Augmented shopping and "Bring your own device": In augmented shopping, the real shopping environment at the POS is additionally extended by a virtual solution (augmented reality). As shown in Figure 1, customers wear augmented reality glasses with which they can virtually put together outfits or change their color selection, but still perceive the real environment. For example, unavailable items at the POS or alternative products are visualized on an avatar in AR glasses. With the help of their visual feedback, customers act as a link between manufacturer and retailer within 
the sales process and thus help retailers to create an assortment that meets their needs. This, in turn, allows production to be better aligned with demand and reduces the need to dispose of excess stock. This process thus leads to a new form of traceability of customer wishes. In a further expansion stage, the garments are inte- grated into apps on mobile devices ("bring your own device") and visualized on the customer's own morphotype. This means that customers can view the fit of a garment on their "digital body" in the app without having to go to the fitting room to try it on, as was previously the case.



Figure 1: Color selection with augmented reality glasses (Source: TH Köln).

B2B - Virtual retailer feedback: In this scenario, apparel retailers exchange ideas with manufacturers about future collections in the same virtual space (Figure 2), instead of the usual location-based approach with multiple meetings. This is done with the help of virtual reality glasses, which allow retailers and manufacturers to examine virtual prototypes as realistically as possible. This leads to location-independent work, saves long journeys and in turn supports intensive coordination and communication processes between dealers and manufacturers. Buyers and designers in particular serve as a link between manufacturer and dealer during prototype development. Savings on prototypes mean that design and prototype development activities are shifting away from 2D processing to 3D simulation. Designers, who previously worked haptically with physical models, are thus dealing exclusively digitally.

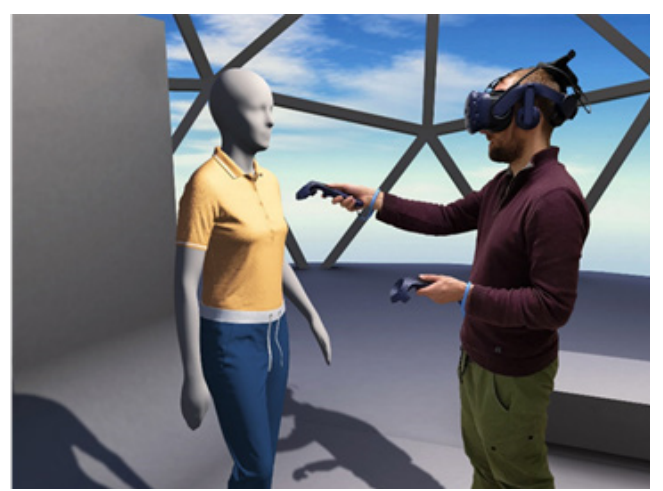

Figure 2: Feedback on the collection with the help of virtual reality glasses (Source: TH Köln).

The solutions enable apparel retailers to exert significant influence on their future assortment at an early stage of development and to provide customers with a unique shopping experience. In particular, the mechanisms for user-community interaction lead to the exploitation of new creative potential, increased customer loyalty and thus significant competitive advantages.

\section{Outlook: Integration into a Digital Development Process}

The aim of the Retail 4.0 research project was to develop a modular, scalable software solution that enables a prompt transfer of feedback from retailers on collection development to the manufacturer and directly involves the customer on the basis of a new, digital VR/AR shopping experience. The tools, that were explored in the project, can also be used along the entire development and production process in the textile industry. In the project "Digital Collection Development", the successive transformation of digitalization in different phases of collection development was investigated. Compared to a radical complete changeover to digital tools, it promises rapid utilization of digitization potential with manageable effort. But where do you start with a successive transformation? Where in the company is the greatest potential for digitization? And how 
must digitized areas in the collection development process be designed to get the most out of digitization? These questions will be answered as part of the project, which is still ongoing. Since every company is different, their potentials are also very individual. A method for individually determining the digitization potential of companies is therefore to be developed. Material flow cost analysis models [12], which are created for the respective collection developments and digitization approaches, serve this purpose.

\section{Conclusion}

This article describes the problems and challenges that arise in the course of digital transformation in the apparel industry as well as the state of the art of the available technologies in the retail industry. Against this background, the need for new, digital solutions, especially for integrating the customer, manufacturer and retailer in the process, became apparent. The solutions developed in the project were to be offered as a service to the retailer and made available to the end customers. However, due to the corona pandemic that began in the last year of the project, a completely virtual concept was developed for this purpose, enabling location-independent exchange in a virtual showroom. The test environment took place in a laboratory environment at the DITF and as a pilot environment in the development center of the clothing manufacturer accompanying the project. In addition to the numerous challenges, the pandemic also offers potential and opportunities to integrate all participants in the product development process. For companies that previously had no online business, this was the only way to sell their products in times of the crisis. For other companies, which had not previously been appropriately positioned or prepared in the area of digitization, this was now the final push. This also applies to product development, which enables retailers and manufacturers to offer other formats, e.g. VR/AR, which are bilateral or digital and independent of location, thus avoiding long journeys and coordination processes.

Due to increasing digitalization, small and medium-sized companies in the textile industry in particular, are under pressure and need integrated, interactive solutions in order to stand out from the competition through individualized service offers. The communication solutions and software developed are conceptually transferable and can be applied to future R\&D projects in the long term. This will lead to enormous savings in product development time, costs and production of sample parts and their presentation through elaborate photo shoots and will mean a clear competitive advantage for the German textile and clothing industry. In addition, the solutions show how customers can influence the collection development with the help of such digital interaction systems and thus become part of the development process.

Based on the results of the project, there is still a need for research that can be addressed in further projects. These include, in particular, virtualization in the individual knowledge-intensive process stages, i.e. digital and virtual developments of all phases involved in the process and their integration into the overall process.
It is to be expected that completely new fields of application will also emerge on this basis.

Furthermore, new, disruptive business models are already emerging for the industry. An example of this is the concept of micro factories for the production of clothing close to the retail pointof-sale. Such a micro factory has already been shown as a demonstrator together with the Retail 4.0 technology components for VR/ AR (13).

\section{Acknowledgements}

We would like to thank the Project Management Agency Karlsruhe (PTKA) for the support and the Federal Ministry of Education and Research (BMBF) for funding the research programme "Retail 4.0 - Virtual Retailer feedback and augmented shopping as a knowledge-intensive, digital service in the development and sales process "(funding code 02K16C231).

In addition, we would like to thank the German Federation of Industrial Research Associations "Otto von Guericke" eV (AiF) for the financial support of the research program "Digital collection development - Selective digitization of collection development processes in the clothing industry on the basis of company-specific potential analyzes using parameterizable MFCA models" (IGF project number: $20892 \mathrm{~N}$ ), which was funded by the Federal Ministry of Economics and Technology (BMWi).

\section{Conflict of Interest}

Authors declare no conflict of interest.

\section{References}

1. Moltenbrey F, Tilebein M (2020) Potentials and challenges of new digital interaction systems in the collection development process of the clothing industry. Freitag M (Editor), Human-Technology Interaction in the Digitized Working World. GITO Verlag, Berlin, Germany, pp. 59-78.

2. Heine U (2018) Medium-sized textile businesses are going digital. TEXTILPLUS (03/04), p. 06-09.

3. Tagesspiegel (2011) Production cycles. Always faster and newer.

4. Retail 4.0 final report will be made publicly available.

5. (2017) The virtual world is accessible to small businesses (2017).

6. Bruns M (2015) Virtual Reality: An Analysis of Key Technology from the Perspective of Strategic Management. Diplomica Verlag, Hamburg, Germany

7. (2017) Trend study: The future of stationary retail

8. Jüngling T (2017) Ikea app projects furniture into your own apartment.

9. Volvocars (2017) Microsoft Hololens.

10. Langbehn A (2010) Practical Guide to Product Development. Basics, tools and examples. Campus, Frankfurt / New York, USA.

11. Henkel R (2018) Faster Fashion: Digitization will radically change the fashion industry.

12. Becker J, Rosemann M, Schütte, R (1995) Principles of Correct Modeling Business informatics 37(5): 435-445.

13. Messe Stuttgart (2021) TecCheck Area. 